\title{
Predicting Menstrual Recovery After Laparoscopic Sleeve Gastrectomy in Polycystic Ovary Syndrome Women With Obesity
}

\section{Meili Cai}

Tongji University Tenth People's Hospital: Shanghai Tenth People's Hospital

\section{Yuqin Zhang}

Tongji University Tenth People's Hospital: Shanghai Tenth People's Hospital Jingyang Gao

Tongji University Tenth People's Hospital: Shanghai Tenth People's Hospital

\section{Diliqingna Dilimulati}

Tongji University Tenth People's Hospital: Shanghai Tenth People's Hospital

\section{Ziwei Lin}

Tongji University Tenth People's Hospital: Shanghai Tenth People's Hospital

\section{Le Bu}

Tongji University Tenth People's Hospital: Shanghai Tenth People's Hospital

\section{Xiaoyun Cheng}

Tongji University Tenth People's Hospital: Shanghai Tenth People's Hospital

\section{Lei Du}

Tongji University Tenth People's Hospital: Shanghai Tenth People's Hospital

Donglei Zhou

Tongji University Tenth People's Hospital: Shanghai Tenth People's Hospital Jiangfan Zhu

Tongji University Tenth People's Hospital: Shanghai Tenth People's Hospital

Shen Qu

Tongji University Tenth People's Hospital: Shanghai Tenth People's Hospital

Manna Zhang ( $\nabla$ mannazhang@126.com )

Shanghai Tenth People's Hospital https://orcid.org/0000-0002-8445-1954

\section{Research}

Keywords: Polycystic ovary syndrome, Obesity, Laparoscopic sleeve gastrectomy, Menstruation

Posted Date: September 7th, 2021

DOI: https://doi.org/10.21203/rs.3.rs-861231/v1 
License: (c) (i) This work is licensed under a Creative Commons Attribution 4.0 International License. Read Full License 


\section{Abstract}

Objectives: To investigate the recovery of menses in polycystic ovary syndrome (PCOS) women with obesity after laparoscopic sleeve gastrectomy (LSG), and to explore the predictors for menstrual recovery.

Methods: A total of 88 PCOS patients with obesity and 76 control patients with obesity aged 18-45 years were enrolled between May 2013 and December 2020. Preoperative clinical characteristics, metabolic parameters and sex hormones were collected. Postoperative menstrual status, body weight and use of oral contraceptives were obtained through telephone follow-up.

Results: Patients with PCOS were followed up for at least six months after surgery, and the mean followup time was 3.23 years. About $24.66 \%$ of patients with PCOS started their first menstruation after LSG within 7 days, and $79.52 \%$ patients had regular menstruation at six months after LSG. In logistic regression analyses, duration of PCOS $\leq 4.5$ years $(P=0.007)$, number of preoperative menstruation per year $>4.0$ times/year $(P=0.045)$, homeostatic model assessment of insulin resistance (HOMA-IR) $\leq 7.3$ $(P=0.020)$ and total testosterone $(T T) \leq 1.5 \mathrm{nmol} / \mathrm{L}(P=0.029)$ at baseline were significantly associated with menstrual resumption in PCOS patients within 6 months after LSG. After adjusting confounders, combination of four clinical indices at baseline could effectively predict menstrual recovery in PCOS patients within 6 months after LSG (AUC=0.837, [95\%Cl: 0.735-0.939], $P<0.001$ ).

Conclusion: Overall, the combination of PCOS duration, menstruations per year, HOMA-IR and TT at baseline could be used as an effective tool for the prediction of menstrual recovery in PCOS patients within 6 months after LSG, which can be applied in preoperative evaluation.

\section{Introduction}

Polycystic ovary syndrome (PCOS) is a common disease that causes ovulatory dysfunction and infertility in reproductive-aged women, and characterized by oligo-amenorrhea, hyperandrogenism, and the presence of polycystic ovaries. Moreover, PCOS women are predisposed to metabolic syndrome, and nearly half of the PCOS women are overweight or obese [ $\left.{ }^{1}\right]$. Studies have found obesity amplifies and worsens all metabolic and reproductive outcomes in PCOS [ $\left.{ }^{2}\right]$. Therefore, bariatric surgery, especially laparoscopic sleeve gastrectomy (LSG), has become the effective therapy for PCOS women with obesity. Except for modification of metabolic and hormonal indicators, bariatric surgery also improves clinical symptoms of PCOS patients within a short period, and increases pregnancy and fertility rates $\left.{ }^{3}\right]$.

At present, the etiology of PCOS remains unclear, and PCOS is mainly treated by symptomatic therapies, including oral contraceptive pills, antiandrogen agents, insulin-sensitizing drugs, lifestyle modification and bariatric surgery. For PCOS women, recovery of menses is not only a vital symptom of improved ovulation function but also an important sign for the effectiveness of the treatment. Meanwhile, regular menstruation is an important indicator of female reproductive system health and endocrine condition [ $\left.{ }^{4}\right]$. Previous studies have demonstrated that irregular and long menstrual cycles are associated with a higher 
risk of type 2 diabetes (T2DM) and coronary heart disease compared with women who had a regular cycle $[5,6,7]$. Thus, it is important to investigate the changes in the menstrual status of PCOS women with obesity after bariatric surgery. Multiple studies have shown that bariatric surgery could restore the regularity of menstrual cycles, ameliorate ovulatory dysfunction and adjust the length of the follicular phase in women with obesity $[8,9,10]$. However, few studies are available on menstrual recovery in PCOS patients with obesity after LSG $\left[{ }^{11},{ }^{12}\right]$, and clinical factors associated with menstrual recovery in PCOS patients are still unknown or have not been extensively studied. We hypothesize that there are some clinical indices at baseline could prognosis the menstrual status in PCOS patients with obesity after LSG.

Therefore, our study aimed to investigate the changes in the menstrual status of PCOS women with obesity after LSG and to explore the prediction model for regular menstrual cycles in PCOS women with obesity after LSG. The study will help the clinicians to assess and understand the prognosis of PCOS patients after LSG in terms of restored menstruation.

\section{Methods}

\subsection{Study Design and Patients}

This study enrolled 88 PCOS women with obesity and 76 age- and body mass index (BMI)-matched subjects with normal menstrual cycle who were admitted to the Department of Endocrinology, Shanghai Tenth People's Hospital, between May 2013 and December 2020 (Fig. 1). All patients were aged 18-45 years and underwent the first operation of LSG. Diagnosis of PCOS was based on the Rotterdam diagnosis criteria (2003) $\left[{ }^{13}\right]$. Exclusion criteria were as follows: 1 ) age $<18$ years or $>45$ years; 2 ) secondary obesity due to endocrine disorders; 3 ) history of more than one bariatric procedure; 4) severe hepatic, renal dysfunction and/or heart failure; and 5) mental illnesses that rendered them unable to provide informed consent. The control patients had regular menstrual cycles (25-35 days) without any clinical and/or biochemical signs of hyperandrogenism. Irregular menstrual cycles were defined as menstruations occurring outside the interval of 25 to 35 days reported by patients, and/or oral contraceptive use to regulate periods. [ $\left.{ }^{14}\right]$.

\subsection{Anthropometric and Telephone Follow-up}

Anthropometric measurements including body weight, height, systolic blood pressure, diastolic blood pressure, waist circumference (WC) and hip circumference (HC) were collected by trained endocrinology specialists from all patients. The number of menstruations per year was defined as the absolute number of menstruations during one year. BMI was calculated as the body weight $(\mathrm{kg})$ divided by the square of the body height $\left(\mathrm{m}^{2}\right)$. The waist to hip ratio (WHR) were calculated was calculated by $\mathrm{HC}$ in centimeters divided by WC in centimeters. Preoperative hirsutism is assessed by the Ferriman-Gallwey score in each of nine locations of the body by two trained endocrinology specialists (including upper lip, chin, chest, upper back, lower back, upper abdomen, lower abdomen, upper arms and thighs) [ $\left.{ }^{15}\right]$. Dual-energy X-ray absorptiometry (DEXA) measurements offer a quick noninvasive estimation of total body \%fat, android 
fat mass and visceral adipose tissue (VAT) mass. The abdominal subcutaneous adipose tissue (SAT) mass was calculated as android fat mass minus VAT mass [ $\left.{ }^{16}\right]$. Postoperative menstrual status, BMI and use of oral contraceptives in PCOS women with obesity were obtained through telephone follow-up after surgery. Menstrual regularity within 6 months after LSG was defined as the patient self-reported having consecutive spontaneous ( $\geq 2$ times) menstrual cycles (25-35 days) within 6 months and without taking oral contraceptives, which continues to exist until the final follow-up time.

\subsection{Blood chemistry}

Blood samples were obtained from all patients in the morning after at least 10 hours of overnight fasting. Fasting plasma glucose (FBG), fasting serum insulin (FINS), glycosylated hemoglobin (HbA1c), uric acid (UA), creatinine, alanine aminotransferase (ALT), aspartate aminotransferase (AST), total cholesterol (TC), triglycerides (TG), high-density lipoprotein cholesterol (HDL-C), and low-density lipoprotein cholesterol (LDL-C), superoxide dismutase (SOD), interleukin-6 (IL-6), interleukin-8 (IL-8), and sex hormone binding globulin (SHBG) were measured. Postprandial plasma glucose (PBG) and postprandial insulin (PINS) were performed using the standard oral glucose tolerance test, followed by examination at 120 min $\left[{ }^{17}\right]$. Luteinizing hormone (LH), follicle-stimulating hormone (FSH), and total testosterone (TT) were measured using an immunochemiluminometric assay (Roche Diagnostics $\mathrm{GmbH}$, Cot., Sandhofer, Mannheim, Germany). Calculated free testosterone (cFT) was calculated with the formula $=24.00314 \times[\mathrm{TT}] / \log 10[\mathrm{SHBG}] 0.04599 \times[\mathrm{TT}]^{2}\left[{ }^{18}\right]$. Homeostatic model assessment of insulin resistance (HOMA-IR) was calculated using the formula=[FBG (mmol/L)×FINS (mU/L)]/22.5 [ $\left.{ }^{19}\right]$. The area under curve (AUC) of insulin was calculated with the following formula: AUC-insulin=(insulin 0 min+insulin $30 \mathrm{~min}$ ) $\times($ insulin $30 \mathrm{~min}+$ insulin $120 \mathrm{~min}$ )×90/2+(insulin $120 \mathrm{~min}+$ insulin $180 \mathrm{~min}$ )×60/2 $\left[{ }^{20}\right]$. The percentage excess weight loss $(\% \mathrm{EWL})$ was calculated as follows: $\% \mathrm{EWL}=100 \% \times$ (baseline weight-last weight)/(baseline weight-ideal weight).

\subsection{Statistical Analysis}

All statistical analyses were analyzed using SPSS Statistics (IBM SPSS Statistics, Version 25.0, 2017, IBM Corp, Armonk, NY) and GraphPad Prism 8 (GraphPad Software, San Diego, USA). All normal distribution continuous data were presented as means \pm standard deviation (SD). Categorical variables were presented as percentages. Continuous data were compared between the two groups using an independent sample t test. Qualitative variables were analyzed with chi-square test. Logistic regression analysis was performed to explore the prognostic factors and to create the new combined predictive indicator that could predict the recovery of regular menstrual cycles within 6 months after LSG in the PCOS group. All independent variables were firstly transformed into dichotomous categorical variables based on the median or clinical cut-off value. The receiver operating characteristic (ROC) curve for the new combined predictive indicator was built for evaluating the recovery of regular menstrual cycles within 6 months after LSG. The optimal cut-off values were identified by the area under the curve (AUC) and the Youden index analysis. According to the optimal cut-off point, PCOS women with obesity were categorized into low probability and high probability groups. Diagnostic parameters such as sensitivity 
and specificity were also calculated. A Kaplan-Meier curve was generated in each probability group. A value of $P<0.05$ was considered as statistically significant.

\section{Results}

\subsection{Comparison of Clinical Characteristics, Sex Hormone and Body Fat Distribution in Obese Women with or without PCOS at Baseline}

As shown in Fig.1, 164 female individuals with obesity were recruited in the study, including 88 PCOS patients and 76 control patients. As presented in Table 1, participants of this study in the PCOS and control groups were relatively young $(29.0 \pm 5.4$ vs $30.1 \pm 7.1$ years). Although the mean BMI and the mean percentage of total body fat before surgery were comparable in two groups, the mean WC, WHR, and VAT mass in the PCOS group were higher than the control group $(P<0.05)$. The mean number of menstruations per year and the levels of HDL-C at baseline in the PCOS group were significantly lower than the control group $(P<0.05)$. Moreover, the mean levels of HOMA-IR, FINS, PINS, AUC-insulin, UA, ALT, AST and TG in the PCOS group were higher than the control group $(P<0.05)$. Compared with the control group, the mean plasma levels of TT and CFT were higher in the PCOS group $(P<0.05)$. Whereas, the mean plasma levels of LH, FSH, SHBG between the two groups were not significantly different $(P>0.05)$.

\subsection{The Menstrual Status, BMI and Use of Oral Contraceptives of PCOS Women with Obesity after LSG}

All patients in the PCOS group were at least six months after surgery when followed up by telephone, and the mean follow-up time was 3.23 years (range, 0.61-7.95 years). As detailed in Figure 2, there was significant improvement in the number of preoperative menstruations per year (4.7 \pm 2.7 vs $10.6 \pm 2.1$; $P<0.001)$ after LSG. Usage of oral contraceptive significantly decreased from $57.95 \%(n=51 / 88)$ preoperatively to $15.91 \%(n=14 / 88 ; P<0.05)$ at the final follow-up of post-surgery. Moreover, the proportion of regular menstruation in PCOS patients was significantly increased within one month $(40.7 \%)$ and six months $(79.52 \%)$ after LSG $(P<0.05)$. Strikingly, $24.66 \%$ of the patients in the PCOS group started their first menstruation after surgery within 7 days. Besides, the mean \%EWL at 1 month, 6 months and the final follow-up after surgery was reached $22.95 \pm 11.4 \%, 67.85 \pm 20.62 \%$ and $79.16 \pm 25.81 \%$, respectively.

\subsection{The Predictive Model for the Regular Menstruation in PCOS Women with Obesity after LSG}

In our study, 79.52\% of patients with PCOS restored their regular menstruation 6 months after LSG, as presented in Figure 2. Thus, recovery of menstruation within 6 months after LSG was defined as an event in the logistic regression analysis. After adjusting for confounders, increased duration of PCOS (adjusted $\mathrm{OR}=0.199 ; 95 \% \mathrm{Cl}: 0.061-0.646, P=0.007)$, reduced number of preoperative menstruations per year (adjusted OR=3.539; 95\% Cl:1.030-12.153, $P=0.045$ ), increased levels of HOMA-IR (adjusted OR=0.237; 95\% Cl: 0.070-0.799, $P=0.020$ ), increased FINS (adjusted OR=0.230; 95\% Cl: 0.067-0.783, $P=0.019$ ), increased TT (adjusted OR=0.241; 95\% Cl: 0.067-0.867, $P=0.029$ ) and $\mathrm{cFT}$ (adjusted OR=0.013; 95\% Cl: $0.001-0.615, P=0.013)$ at baseline were demonstrated to be independent risk factors for the regular menstruation in PCOS women with obesity within 6 months after LSG in the logistic equation. However, 
there was no statistical correlation between other clinical indicators (including BMI, HbA1c, lipid profile, $\mathrm{UA}, \mathrm{LH}, \mathrm{FSH}, \mathrm{SHBG}$, and body fat composition) and regular menstruation status (Table 2).

The predictive model combined four preoperative clinical indices in terms of categorical covariates, including duration of PCOS, number of preoperative menstruations per year, HOMA-IR and TT (cut-off values was $4.5,4.0,7.3$, and 1.5 , respectively), could predict the recovery of menstruation in PCOS women with obesity within 6 months after LSG (AUC=0.837, [95\% Cl: 0.735-0.939], $P<0.001)$. When using the combined predictive indicator to predict the regular menstruation in PCOS women with obesity within 6 months after LSG, the sensitivity and specificity were $66.7 \%$ and $88.9 \%$. Meanwhile, according to the optimal cut-off point (the maximal values of the Youden index was 0.556 ) in the adjusted model, PCOS women with obesity were categorized into the high probability group and the low probability group. Kaplan-Meier curves showed that compared with the low probability group, the high probability group was more than 2 times likely to recovery menstruation [HR=2.543, (95\% Cl: 1.147-5.638), $P=0.022$ ]. (Figure 3)

\section{Discussion}

PCOS is a common reproductive endocrine disease, and the majority of PCOS patients have different degrees of insulin resistance, chronic inflammation, T2DM, lipids dysfunction [1,2], all of which are exacerbated with the presence of obesity. As a specific manifestation of ovarian dysfunction, the recovery of the menstrual cycle is the main goal of PCOS management in clinical, and also the main appeal of PCOS patients with obesity. Multiple observational studies have reported that LSG could improve hormone profile and fertility outcome in patients with PCOS [21, 22]. However, limited studies were available on the prognostic of the menstrual status in PCOS patients with obesity after LSG. Our study is the first to explore the predictive factors for regular menstruation in PCOS women with obesity after LSG.

In our study, although the mean age and BMI were comparable in the PCOS group and the control group, the PCOS group had higher WC, WHR, and VAT mass. The findings were generally in line with previous studies that PCOS patients tend to be more centrally obese than non-PCOS patients $\left[{ }^{23},{ }^{24}\right]$. In addition, we found that PCOS patients with obesity had higher UA, severer insulin resistance and lipids dysfunction than regular menstruation patients with obesity at baseline, which is consistent with previous studies that PCOS patients with obesity demonstrate more severe metabolic abnormalities $[25,26,27]$. However, there were no differences in the levels of IL- 6 and SOD between the PCOS group and the control group. Conversely, some studies reported that IL- 6 levels and SOD activity increased in the patients with PCOS $\left[{ }^{28},{ }^{29}\right]$. The discrepancy might be explained by different populations of studies (age, ethnicity, body weight, and duration of PCOS). Meanwhile, we also found that PCOS women with obesity exhibited increased levels of TT and cFT than the control groups, which is in lined with previous population-based studies $\left[{ }^{30}, 31\right]$. In addition, other sex hormones of PCOS patients with obesity, including LH, FSH and SHBG were not statistically significant different compared with the control group in our study, which is 
inconsistent with other studies $\left[{ }^{32}, 33\right]$. However, this result needs to be validated in studies with a larger sample size.

To our knowledge, regular menstruation is determined by a dynamic reproductive endocrine system, including ovarian events, hypothalamic-pituitary interactions, and changes in the endometrium and myometrium [ $\left.{ }^{34}\right]$. Therefore, menstrual recovery is an easily ascertainable external parameter for the improvement of ovarian function. Moreover, the regularity of menstruation is the main demand of PCOS patients with obesity. Thus, it is important to explore the menstrual status in PCOS women with obesity after LSG.

In the present study, we followed-up the menstrual status in the PCOS group via telephone interview, and the median follow-up time was 3.07 years. Our data showed that $75.86 \%$ of PCOS patients with obesity could recover regular menstrual cycles within 6 months after LSG. In line with our study, a meta-analysis found that patients with PCOS after surgery had a significantly high incidence of having normal menstruation [ $\left.{ }^{35}\right]$. Damhof et al. has reported that there was significant, nearly 2-fold, reduction of oral contraceptives in women after bariatric surgery [ $\left.{ }^{36}\right]$. Consistent with this research, our data showed that the use of oral contraceptives in PCOS women after LSG was significantly decreased after the operation, which supported the fact of menstrual recovery in these patients. It was interesting to note that $24.66 \%$ of patients in the PCOS group started their first menstruation after LSG within 7 days. However, the underlying mechanism of this phenomenon was unclear, which might be attributed to the changes in gastrointestinal hormones, insulin resistance, and hypothalamic-pituitary-adrenal axis right after LSG [ ${ }^{37}$ ]. Further studies are needed to address this issue.

Our further analyses were performed to explore the factors that may predict the regularity of menstrual cycles in PCOS patients after LSG. Our results showed duration of PCOS ( $\leq 4.5$ years), number of preoperative menstruations per year (>4.0 times/years) levels of HOMA-IR $(\leq 7.3)$, and TT $(\leq 1.5 \mathrm{nmol} / \mathrm{L})$ were useful predictors for the regularity of menstrual cycles in PCOS patients with obesity within 6 months after LSG. The findings suggested that the resumption of menses in PCOS women with obesity after LSG was closely associated with the duration of PCOS and previous menstrual status. Moreover, growing evidence has demonstrated that the menstrual cycle abnormality appeared to be associated with more pronounced insulin resistance and hyperandrogenemia in PCOS [ $\left.{ }^{38},{ }^{39}\right]$, as insulin resistance can induce oligo- or anovulation and cause menstrual cycle irregularity by exacerbating hyperandrogenemia and disrupting follicular growth $[2,40]$. In addition, previous studies showed that hyperandrogenemia was significantly associated with long and irregular menstruation in women with PCOS $\left[{ }^{41}\right]$. Ovarian hyperandrogenism can inhibit folliculogenesis and lead to poly-follicular morphology, which can disrupt the menstrual cycle. Overall, it is suggested that serum TT and HOMA-IR levels may be used as predictors for the recovery of the menstrual cycle after LSG.

Consistent with our research, Ran $Y$ et al. have reported that the components of blood lipids were not statistically significant between the menstrual regularity group and the menstrual disorder group [ $\left.{ }^{42}\right]$, as a 
result, changes in lipid metabolism might not be the main cause of the improvement of menstruation in PCOS patients with obesity after LSG. Besides, there was no correlation between other clinical features (especially BMI, $\mathrm{HbA} 1 \mathrm{c}, \mathrm{UA}$, and body fat composition) and the recovery of the menstrual cycles in PCOS patients with obesity after LSG in our study, although some studies have reported that the recovery degree of metabolic syndrome after bariatric surgery can also be used as one of the evaluation criteria for the improvement of clinical efficacy of PCOS patients with obesity $\left[{ }^{43}, 44\right]$. Among sex hormones, the regular menstruation within 6 months after surgery in women with PCOS was not correlated with levels of $\mathrm{LH}, \mathrm{FSH}$ and SHBG at baseline. Therefore, those clinical indicators may have a limited effect on the recovery of menstruation after LSG.

Furthermore, we formed a combined model with the four clinical indices at baseline (including duration of PCOS, number of preoperative menstruations per year, HOMA-IR and TT) to predict the recovery of menstruation after LSG, and the area under the ROC curve of the combined model is 0.837 [95\% Cl: 0.735 0.939]. Additionally, we further categorized patients into low probability and high probability groups according to the optimal cut-off point. Compared with the low probability group, those in the high probability group had 2.5-fold increases in the odds of the resumption of menses within 6 months after LSG. Thus, the combined model is fairly accurate in predicting the regular menstruation in PCOS women with obesity within 6 months after LSG.

The limitations in this study were listed as follows: first, our study was a retrospective study and menstrual status was self-reported by patients, which might result in recall bias; second, telephone followup to assess menstrual recovery may lack of accuracy than gynecological ultrasound. Third, the prediction model has not been verified in other populations and future studies need to involve a larger population of PCOS women with obesity to confirm the accuracy of this prediction model.

\section{Conclusions}

In the Chinese population of PCOS women with obesity, the frequency of regular menstrual cycles could be greatly improved after LSG. Duration of PCOS ( $\leq 4.5$ years), number of preoperative menstruations per year (> 4.0 times/year), levels of HOMA-IR $(\leq 7.3)$ and TT $(\leq 1.5 \mathrm{nmol} / \mathrm{L})$ at baseline are useful predictors for the regularity of menstrual cycles in PCOS patients with obesity within 6 months after LSG. This study suggested that the combination of those four clinical indices could be used as an inexpensive and convenient tool for early prediction of regularity menstruation among PCOS patients with obesity after LSG.

\section{Abbreviations}

LSG: laparoscopic sleeve gastrectomy; PCOS: Polycystic ovary syndrome; T2DM: Type 2 diabetes; BMI: body mass index; WC: waist circumference; HC: Hip circumference; WHR: Waist to hip ratio; DEXA: Dualenergy X-ray absorptiometry; VAT: Visceral adipose tissue; SAT: The abdominal subcutaneous adipose tissue; FBG: Fasting plasma glucose; FINS: Fasting serum insulin; HbA1c: Glycosylated hemoglobin; UA: 
Uric acid; ALT: Alanine aminotransferase; AST: Aspartate aminotransferase; TC: Total cholesterol; TG: Triglycerides; HDL-C: High-density lipoprotein cholesterol; LDL-C: Low-density lipoprotein cholesterol; SOD: Superoxide dismutase; IL-6: Interleukin-6; IL-8: Interleukin-8; SHBG: sex hormone binding globulin; PBG: Postprandial plasma glucose; PINS: postprandial insulin; LH: Luteinizing hormone; FSH: Folliclestimulating hormone; TT: Total testosterone; $\mathrm{CFT}$ : Calculated free testosterone; \%EWL: The percentage excess weight loss; HOMA-IR: Homeostatic model assessment of insulin resistance; ROC: Receiver operating characteristic; AUC: Area under the curve.

\section{Declarations}

\section{Ethical Approval and Consent to participate}

This study was approved by the ethics committee of Shanghai Tenth People's Hospital. Written informed consent was obtained from all participants.

\section{Acknowledgments}

This study would not have been possible without the participation of these patients.

\section{Authors' Contributions}

Meili Cai, Yuqin Zhang and Jingyang Gao: acquisition, telephone follow-up PCOS patients, and statistical analysis; Diliqingna Dilimulati, Ziwei Lin, Bu le and Xiaoyun Cheng: checking and interpretation of the data; Lei Du, Donglei Zhou and Jiangfan Zhu: evaluation and implementation of surgery; Shen Qu and Manna Zhang: design and drafting of the manuscript; All authors reviewed or approved the final version of the manuscript.

\section{Funding}

This study is supported by grants from the National Key R\&D Program of China (No.2018YFC1314100) and the National Nature Science Foundation (No.81601269).

\section{Availability of supporting data}

All data generated or analyzed during this study are included in this published article.

\section{Consent for publication}

The patients signed informed consent regarding publishing their data.

\section{Competing Interests}

The authors declare that they have no competing interests. 


\section{References}

1. Anagnostis $P$, Tarlatzis BC, Kauffman RP. Polycystic ovarian syndrome (PCOS): Long-term metabolic consequences. Metabolism. 2018; 86:33-43.

2. Glueck CJ, Goldenberg N. Characteristics of obesity in polycystic ovary syndrome: Etiology, treatment, and genetics. Metabolism. 2019; 92:108-120.

3. Christ JP, Falcone T. Bariatric Surgery Improves Hyperandrogenism, Menstrual Irregularities, and Metabolic Dysfunction Among Women with Polycystic Ovary Syndrome (PCOS). Obes Surg. 2018;28(8):2171-2177.

4. Messinis IE, Messini Cl, Dafopoulos K. Novel aspects of the endocrinology of the menstrual cycle. Reprod Biomed Online. 2014;28(6): 714-22.

5. Rostami Dovom M, Ramezani Tehrani F, Djalalinia S, Cheraghi L, Behboudi Gandavani S, Azizi F. Menstrual Cycle Irregularity and Metabolic Disorders: A Population-Based Prospective Study. PLoS One. 2016;11(12): e0168402.

6. Shahla Nader. Reproductive endocrinology: menstrual cycle lengths-what can they tell us? Nat Rev Endocrinol. 2012;8(12):704-6.

7. Solomon CG, Hu FB, Dunaif A, Rich-Edwards J, Willett WC, Hunter DJ, et al. Long or highly irregular menstrual cycles as a marker for risk of type 2 diabetes mellitus. JAMA. 2001;286(19):2421-6.

8. Różańska-Walędziak A, Bartnik P, Kacperczyk-Bartnik J, Czajkowski K, Walędziak M. The Impact of Bariatric Surgery on Menstrual Abnormalities-a Cross-Sectional Study. Obes Surg. 2020;30(11):45054509.

9. Teitelman M, Grotegut CA, Williams NN, Lewis JD. The impact of bariatric surgery on menstrual patterns. Obes Surg. 2006;16(11):1457-63.

10. Soares Júnior JM, Lobel A, Ejzenberg D, Serafıni PC, Baracat EC. Bariatric surgery in infertile women with morbid obesity: defınitive solution? Rev Assoc Med Bras. 2018;64(7):565-7.

11. Altamimi AF, Alqahtani ZA, Alshiha KA, Almughaiseeb F, Alfayez NF, Alkhatir AA. The Impact of Sleeve Gastrectomy on Females with Polycystic Ovarian Syndrome From 2018 to 2020 in Riyadh, Saudi Arabia: A Prospective Study. Cureus. 2020;12(9): e10284.

12. Różańska-Walędziak A, Bartnik P, Kacperczyk-Bartnik J, Czajkowski K, Walędziak M. The Impact of Bariatric Surgery on Menstrual Abnormalities-a Cross-Sectional Study. Obes Surg. 2020;30(11):45054509.

13. The Rotterdam ESHRE/ASRM-sponsored PCOS consensus workshop group. Revised 2003 consensus on diagnostic criteria and longterm health risks related to polycystic ovary syndrome (PCOS). Hum Reprod. 2004;19(1):41-7.

14. M Mihm, S Gangooly, S Muttukrishna. The normal menstrual cycle in women. Anim Reprod Sci. 2011;124(3-4):229-36.

15. Robert L. Rosenfield. Hirsutism. N Engl J Med. 2005;353: 2578-88. 
16. Dhaliwal R, Shepherd JA, El Ghormli L, et al. Changes in Visceral and Subcutaneous Fat in Youth With Type 2 Diabetes in the TODAY Study. Diabetes Care. 2019;42(8):1549-1559.

17. Yang W, Liu J, Shan Z, Tian H, Zhou Z, Ji Q, et al. Acarbose compared with metformin as initial therapy in patients with newly diagnosed type 2 diabetes: an open-label, non-inferiority randomized trial. Lancet Diabetes. 2014; 2: 46-55.

18. Keelan JA, Mattes E, Tan H, Dinan A, Newnham JP, Whitehouse AJ, et al. Androgen concentrations in umbilical cord blood and their association with maternal, fetal and obstetric factors. PLoS One. 2012;7(8): e42827.

19. Gayoso-Diz P, Otero-Gonzalez A, Rodriguez-Alvarez MX, Gude F, Cadarso-Suarez C, García F, De Francisco A. Insulin resistance index (HOMA-IR) levels in a general adult population: curves percentile by gender and age. The EPIRCE study. Diabetes Res Clin Pract. 2011;94(1):146-55.

20. M M Tai. A mathematical model for the determination of total area under glucose tolerance and other metabolic curves. Diabetes Care. 1994;17(2):152-4.

21. Agostinho S Machado Júnior, Cláudio B L Ribeiro, Fernando Santa-Cruz, Sena BF, Figueiredo JL, Ferraz ÁAB, et al. The Effect of Sleeve Gastrectomy on the Hormonal Profile of Patients with Polycystic Ovary Syndrome. Obes Surg. 2019;29(8):2415-2419.

22. Dilday J, Derickson M, Kuckelman J, Reitz C, Ahnfeldt E, Martin M, et al. Sleeve Gastrectomy for Obesity in Polycystic Ovarian Syndrome: a Pilot Study Evaluating Weight Loss and Fertility Outcomes. Obes Surg. 2019;29(1):93-98.

23. Agnieszka Pazderska, Tommy Kyaw Tun, Niamh Phelan, McGowan A, Sherlock M, Behan L, et al. In women with PCOS, waist circumference is a better surrogate of glucose and lipid metabolism than disease status per se. Clin Endocrinol. 2018;88(4):565-574.

24. Cai M, Gao J, Du L, Cheng X, Zhou D, Zhu J, et al. The Changes in Body Composition in Obese Patients with Polycystic Ovary Syndrome after Laparoscopic Sleeve Gastrectomy: a 12-Month Follow-up. Obes Surg. 2021 Jun 19.

25. Liangshan Mu, Jiexue Pan, Lili Yang, Chen Q, Chen Y, Teng Y, et al. Association between the prevalence of hyperuricemia and reproductive hormones in polycystic ovary syndrome. Reprod Biol Endocrinol. 2018;16(1):104.

26. V Wekker, L van Dammen, A Koning, Heida KY, Painter RC, Limpens J, et al. Long-term cardiometabolic disease risk in women with PCOS: a systematic review and meta-analysis. Hum Reprod Update. 2020;26(6):942-960.

27. Wild RA, Rizzo M, Clifton S, Carmina E. Lipid levels in polycystic ovary syndrome: systematic review and meta-analysis. Fertil Steril. 2011;95(3):1073-9.e1-11.

28. Khashchenko E, Vysokikh M, Uvarova E, Krechetova L, Vtorushina V, Ivanets T, et al. Activation of Systemic Inflammation and Oxidative Stress in Adolescent Girls with Polycystic Ovary Syndrome in Combination with Metabolic Disorders and Excessive Body Weight. J Clin Med. 2020;9(5):1399.

29. Jeelani H, Ganie MA, Masood A, Amin S, Kawa IA, Fatima Q, et al. Assessment of PON1 activity and circulating TF levels in relation to BMI, testosterone, HOMA-IR, HDL-C, LDL-C, CHO, SOD activity and 
TAC in women with PCOS: An observational study. Diabetes Metab Syndr. 2019;13(5):2907-2915.

30. Barrea L, Arnone A, Annunziata G, Muscogiuri G, Laudisio D, Salzano C, et al. Adherence to the Mediterranean Diet, Dietary Patterns and Body Composition in Women with Polycystic Ovary Syndrome (PCOS). Nutrients. 2019;11(10):2278.

31. Song DK, Lee H, Oh JY, Hong YS, Sung YA. FTO Gene Variants Are Associated with PCOS Susceptibility and Hyperandrogenemia in Young Korean Women. Diabetes Metab J. 2014;38(4):30210.

32. Piouka A, Farmakiotis D, Katsikis I, Macut D, Gerou S, Panidis D. Anti-Mullerian hormone levels reflect severity of PCOS but are negatively influenced by obesity: relationship with increased luteinizing hormone levels. Am J Physiol Endocrinol Metab. 2009;296(2): E238-43.

33. Qu X, Donnelly R. Sex Hormone-Binding Globulin (SHBG) as an Early Biomarker and Therapeutic Target in Polycystic Ovary Syndrome. Int J Mol Sci. 2020;21(21):8191.

34. JoAnne S Richards. The Ovarian Cycle. Vitam Horm. 2018; 107:1-25.

35. Li YJ, Han Y, He B. Effects of bariatric surgery on obese polycystic ovary syndrome: a systematic review and meta-analysis. Surg Obes Relat Dis. 2019;15(6):942-950.

36. Damhof MA, Pierik E, Krens LL, Vermeer M, van Det MJ, van Roon EN. Assessment of Contraceptive Counseling and Contraceptive Use in Women After Bariatric Surgery. Obes Surg. 2019;29(12):40294035.

37. R Charlotte Moffett, Violetta Naughton. Emerging role of GIP and related gut hormones in fertility and PCOS. Peptides. 2020; 125:170233.

38. Panidis D, Tziomalos K, Chatzis P, Papadakis E, Delkos D, Tsourdi EA, et al. Association between menstrual cycle irregularities and endocrine and metabolic characteristics of the polycystic ovary syndrome. Eur J Endocrinol. 2013;168(2):145-52.

39. Goodman NF, Cobin RH, Futterweit W, Glueck JS, Legro RS, Carmina E, et al. AMERICAN ASSOCIATION OF CLINICAL ENDOCRINOLOGISTS, AMERICAN COLLEGE OF ENDOCRINOLOGY, AND ANDROGEN EXCESS AND PCOS SOCIETY DISEASE STATE CLINICAL REVIEW: GUIDE TO THE BEST PRACTICES IN THE EVALUATION AND TREATMENT OF POLYCYSTIC OVARY SYNDROME-PART 1. Endocr Pract. 2015;21(11):1291-300.

40. Wang J, Wu D, Guo H, Li M. Hyperandrogenemia and insulin resistance: The chief culprit of polycystic ovary syndrome. Life Sci. 2019; 236:116940.

41. Zhang D, Gao J, Liu X, Qin H, Wu X. Effect of Three Androgen Indexes (FAI, FT, and TT) on Clinical, Biochemical, and Fertility Outcomes in Women with Polycystic Ovary Syndrome. Reprod Sci. 2021;28(3):775-784.

42. Ran Y, Yi Q, Li C. Correlation Between Recovery of Menstrual Cycle and Improvement of Glucose and Lipid Metabolism in Patients with Sleeve Gastrectomy in a Small Chinese Cohort Sample. Diabetes Metab Syndr Obes. 2020;13: 4711-4715.

43. Tian Z, Zhang YC, Wang Y, Chang XH, Zhu HL, Zhao Y. Effects of bariatric surgery on patients with obesity and polycystic ovary syndrome: a meta-analysis. Surg Obes Relat Dis. 2021;17(8):1399- 
1408.

44. Abdalla MA, Deshmukh $\mathrm{H}$, Atkin S, et al. A review of therapeutic options for managing the metabolic aspects of polycystic ovary syndrome. Ther Adv Endocrinol Metab. 2020;11: 2042018820938305.

\section{Tables}


Clinical characteristics, sex hormone and body composition parameters in the PCOS group and control group at baseline.

\begin{tabular}{|c|c|c|c|}
\hline Items & $\begin{array}{l}\text { Control group } \\
(n=76)\end{array}$ & $\begin{array}{l}\text { PCOS group } \\
(n=88)\end{array}$ & P Values \\
\hline Age at surgery (years) & $30.1 \pm 7.1$ & $29.0 \pm 5.4$ & 0.223 \\
\hline Age at menarche (years) & $13.0 \pm 1.3$ & $13.3 \pm 2.4$ & 0.354 \\
\hline Duration of obesity (years) & $10.3 \pm 7.5$ & $11.1 \pm 7.0$ & 0.445 \\
\hline Duration of PCOS (years) & $0.0 \pm 0.0$ & $5.9 \pm 5.5$ & $<0.001 *$ \\
\hline Ferriman-Gallway score & $5.4 \pm 4.1$ & $6.8 \pm 4.0$ & 0.203 \\
\hline Number of menstruations per year (no./yr) & $11.2 \pm 0.8$ & $4.6 \pm 2.7$ & $<0.001 *$ \\
\hline SBP $(\mathrm{mmHg})$ & $134.6 \pm 28.3$ & $132.4 \pm 16.0$ & 0.540 \\
\hline $\mathrm{DBP}(\mathrm{mmHg})$ & $83.5 \pm 19.5$ & $83.9 \pm 13.3$ & 0.876 \\
\hline Waist circumference (cm) & $111.6 \pm 13.2$ & $116.3 \pm 12.8$ & $0.029 *$ \\
\hline Hip circumference (cm) & $119.9 \pm 11.2$ & $121.1 \pm 10.7$ & 0.535 \\
\hline Waist/hip Ratio & $0.9 \pm 0.1$ & $1.0 \pm 0.1$ & $0.005^{*}$ \\
\hline $\mathrm{BMI}\left(\mathrm{kg} / \mathrm{m}^{2} \mathrm{\square}\right.$ & $37.4 \pm 5.8$ & $38.7 \pm 5.5$ & 0.162 \\
\hline Total Body Fat (\%) & $46.6 \pm 3.9$ & $47.3 \pm 3.7$ & 0.248 \\
\hline VAT Mass (kg) & $1.1 \pm 0.3$ & $1.2 \pm 0.3$ & $0.013^{*}$ \\
\hline SAT Mass (kg) & $3.1 \pm 0.9$ & $3.4 \pm 1.1$ & 0.053 \\
\hline HbA1c (\%) & $6.1 \pm 1.6$ & $6.1 \pm 1.5$ & 0.937 \\
\hline HOMA-IR & $6.8 \pm 4.5$ & $9.3 \pm 6.3$ & $0.004^{*}$ \\
\hline FBG (mmol/L) & $6.0 \pm 2.1$ & $6.5 \pm 2.6$ & 0.157 \\
\hline PBG (mmol/L) & $9.1 \pm 4.3$ & $10.2 \pm 4.3$ & 0.119 \\
\hline FINS (mU/L) & $25.4 \pm 14.2$ & $32.3 \pm 17.2$ & $0.007^{*}$ \\
\hline PINS (mU/L) & $116.8 \pm 97.1$ & $170.0 \pm 138.6$ & $0.005^{*}$ \\
\hline AUC-insulin & $17734.2 \pm 11000.8$ & $23608.9 \pm 15228.2$ & $0.007 *$ \\
\hline Urid acid (umol/L) & $371.2 \pm 81.3$ & $406.5 \pm 81.0$ & $0.007 *$ \\
\hline Creatinine (umol/L) & $59.1 \pm 21.9$ & $56.3 \pm 13.8$ & 0.332 \\
\hline ALT (U/L) & $35.2 \pm 29.9$ & $57.7 \pm 40.4$ & $<0.001 *$ \\
\hline
\end{tabular}




\begin{tabular}{|llll|}
\hline AST (U/L) & $26.1 \pm 18.9$ & $37.1 \pm 25.3$ & $0.002^{*}$ \\
\hline SOD (U/ml) & $197.9 \pm 30.3$ & $195.2 \pm 28.8$ & 0.586 \\
\hline Total cholesterol (mmol/L) & $4.5 \pm 0.8$ & $4.6 \pm 0.8$ & 0.412 \\
\hline Triglycerides (mmol/L) & $1.6 \pm 0.9$ & $2.0 \pm 1.5$ & $0.025^{*}$ \\
\hline HDL-c (mmol/L) & $1.1 \pm 0.3$ & $1.0 \pm 0.3$ & $0.036^{*}$ \\
\hline LDL-c (mmol/L) & $2.8 \pm 0.7$ & $2.8 \pm 0.8$ & 0.769 \\
\hline Interleukin-6 (pg/mL) & $10.2 \pm 19.2$ & $11.1 \pm 17.4$ & 0.743 \\
\hline Interleukin-8 (pg/mL) & $272.1 \pm 625.3$ & $284.0 \pm 547.7$ & 0.904 \\
\hline Progesterone (nmol/L) & $6.3 \pm 13.2$ & $3.2 \pm 7.0$ & 0.082 \\
\hline LH (IU/L) & $7.3 \pm 7.3$ & $9.0 \pm 13.8$ & 0.348 \\
\hline FSH (IU/L) & $6.1 \pm 5.4$ & $5.2 \pm 4.2$ & 0.220 \\
\hline Total testosterone (nmol/L) & $1.2 \pm 0.7$ & $1.6 \pm 0.9$ & $0.002^{*}$ \\
\hline cFT (nmol/L) & $4.8 \pm 2.8$ & $11.8 \pm 16.0$ & $0.030^{*}$ \\
\hline SHBG (nmol/L) & $23.8 \pm 14.7$ & $19.0 \pm 11.9$ & 0.114 \\
\hline
\end{tabular}

Abbreviation: $S B P$, systolic blood pressure; $D B P$, diastolic blood pressure; $B M I$, body mass index; $V A T$, visceral adipose tissue; $S A T$, abdominal subcutaneous adipose tissue; $H b A 1 c$, glycosylated hemoglobin A1c; HOMA-IR, homeostatic model assessment insulin resistance index; $F B G$, fasting plasma glucose; $P B G$, postprandial plasma glucose; FINS, fasting serum insulin; PINS, postprandial insulin; AUC-insulin, area under curve-insulin; $A L T$, alanine aminotransferase; $A S T$, aspartate aminotransferase; $S O D$, superoxide dismutase; $H D L-c$, high-density lipoprotein cholesterol; $L D L-C$, low-density lipoprotein cholesterol; $L H$, luteinizing hormone; $F S H$, follicle-stimulating hormone; $C F T$, calculated free testosterone; $S H B G$, sex hormone-binding globulin; * Means $P<0.05$. 
Multivariate logistic regressions between variables at baseline and menstrual status of PCOS patients with obesity within 6 months after LSG

\begin{tabular}{|c|c|c|c|c|}
\hline \multirow[t]{2}{*}{ Prognostic Factor } & \multirow[t]{2}{*}{ Subgroup } & \multicolumn{3}{|c|}{$\begin{array}{l}\text { Regular menstruation within } 6 \text { months } \\
\text { after LSG }\end{array}$} \\
\hline & & OR & $95 \% \mathrm{Cl}$ & P Values \\
\hline Age (years) & $>29.0$ vs $\leq 29.0$ & 0.760 & $0.128-4.512$ & 0.760 \\
\hline Duration of PCOS (years) & $>4.5$ vs $\leq 4.5$ & 0.199 & $0.061-0.646$ & $0.007 *$ \\
\hline Duration of obesity (years) & $>10.0$ vs $\leq 10.0$ & 0.813 & $0.263-2.517$ & 0.720 \\
\hline $\begin{array}{l}\text { Number of menstruations per year } \\
\text { (no./yr) }\end{array}$ & $>4.0$ vs $\leq 4.0$ & 3.539 & $1.030-12.153$ & $0.045^{\star}$ \\
\hline BMI $\left(\mathrm{kg} / \mathrm{m}^{2}\right)$ & $>38.3$ vs $\leq 38.3$ & 6.579 & $0.836-51.750$ & 0.073 \\
\hline Total Body Fat (\%) & $>47.0$ vs $\leq 47.0$ & 0.897 & $0.269-2.992$ & 0.859 \\
\hline VAT Mass (kg) & $>1.2$ vs $\leq 1.2$ & 2.327 & $0.654-8.277$ & 0.192 \\
\hline SAT Mass (kg) & $>3.2$ vs $\leq 3.2$ & 1.533 & $0.415-5.665$ & 0.522 \\
\hline HOMA-IR & $>7.3$ vs $\leq 7.3$ & 0.237 & $0.070-0.799$ & $0.020 *$ \\
\hline FBG (mU/L) & $>5.6$ vs $\leq 5.6$ & 0.813 & $0.283-2.345$ & 0.701 \\
\hline FINS (mU/L) & $>27.5$ vs $\leq 27.5$ & 0.230 & $0.067-0.783$ & $0.019 *$ \\
\hline HbA1c (\%) & $>5.9$ vs $\leq 5.9$ & 0.685 & $0.194-2.424$ & 0.558 \\
\hline Uric acid (umol/L) & $\begin{array}{l}>420.0 \text { vs } \\
\leq 420.0\end{array}$ & 0.768 & $0.258-2.280$ & 0.634 \\
\hline Creatinine(umol/L) & $>56.0$ vs $\leq 56.0$ & 0.444 & $0.145-1.365$ & 0.157 \\
\hline Total cholesterol (mmol/L) & $>5.7$ vs $\leq 5.7$ & 0.263 & $0.049-1.425$ & 0.121 \\
\hline Triglycerides(mmol/L) & $>1.7$ vs $\leq 1.7$ & 0.629 & $0.210-1.881$ & 0.407 \\
\hline HDL-c (mmol/L) & $>1.0$ vs $\leq 1.0$ & 0.449 & $0.146-1.386$ & 0.164 \\
\hline LDL-c (mmol/L) & $>2.8$ vs $\leq 2.8$ & 0.766 & $0.259-2.271$ & 0.631 \\
\hline LH (IU/L) & $>6.8$ vs $\leq 6.8$ & 0.897 & $0.292-2.760$ & 0.850 \\
\hline FSH (IU/L) & $>5.1$ vs $\leq 5.1$ & 0.436 & $0.144-1.322$ & 0.143 \\
\hline Total testosterone (nmol/L) & $>1.5$ vs $\leq 1.5$ & 0.241 & $0.067-0.867$ & $0.029 *$ \\
\hline cFT (nmol/L) & $>6.3$ vs $\leq 6.3$ & 0.013 & $0.001-0.615$ & $0.013^{*}$ \\
\hline SHBG (nmol/L) & $>15.0$ vs $\leq 15.0$ & 2.462 & $0.604-10.040$ & 0.209 \\
\hline
\end{tabular}


Abbreviations: $O R$, odds ratio; $C l$, confidence interval; $B M I$, body mass index; $V A T$, visceral adipose tissue; $S A T$, abdominal subcutaneous adipose tissue; HOMA-IR, homeostatic model assessment insulin resistance index; $F B G$, fasting plasma glucose; FINS, fasting serum insulin; $H b A 1 c$, glycosylated hemoglobin A1c; $H D L-c$, high-density lipoprotein cholesterol; $L D L-c$, low-density lipoprotein cholesterol; $L H$, luteinizing hormone; $F S H$, follicle-stimulating hormone; $C F T$, calculated free testosterone; $S H B G$, sex hormone-binding globulin; All data were adjusted for age, BMI, and alanine aminotransferase. * Means $P<0.05$.

\section{Figures}

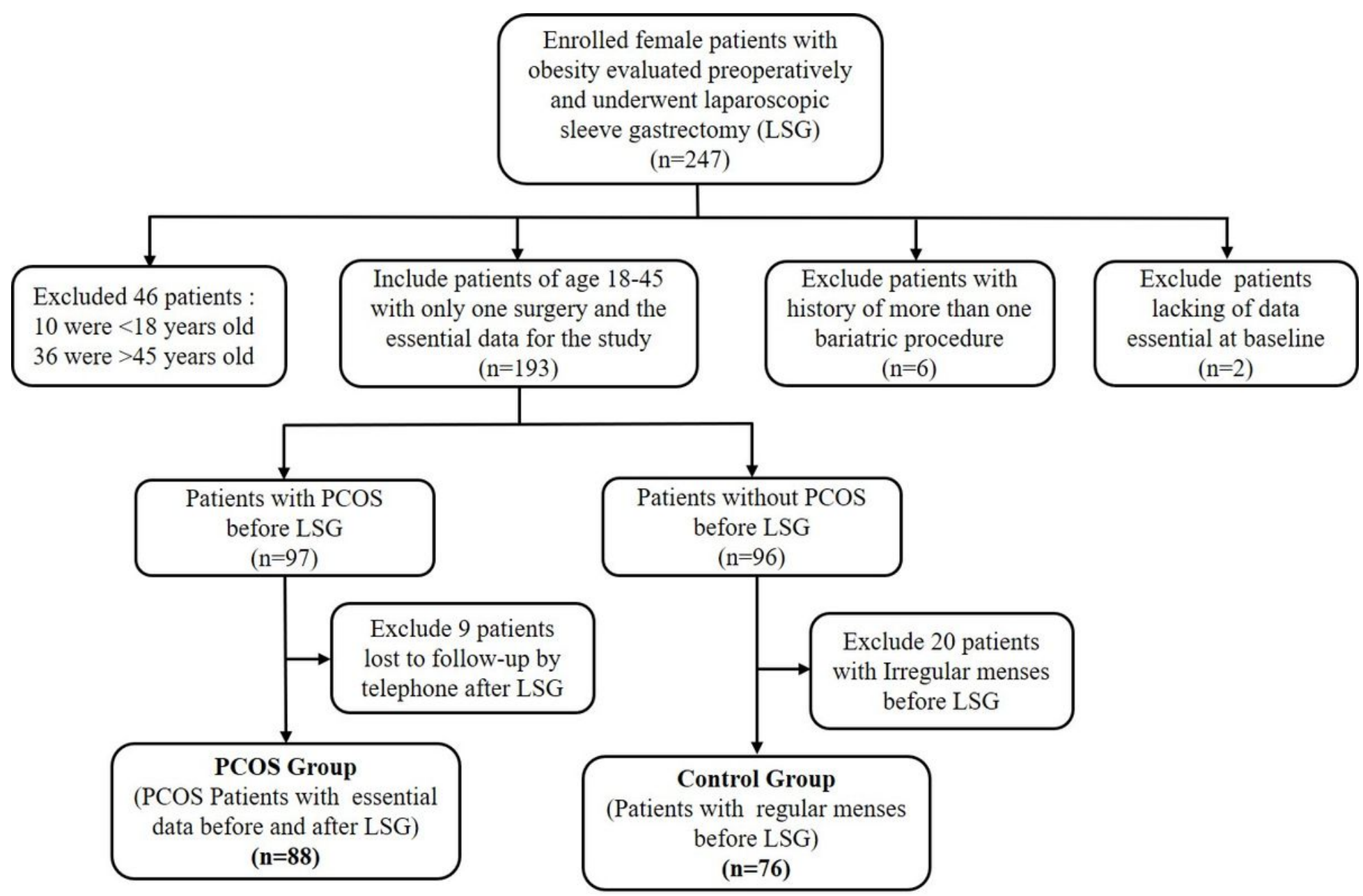

\section{Figure 1}

The flow diagram of patient recruitment and grouping process. 


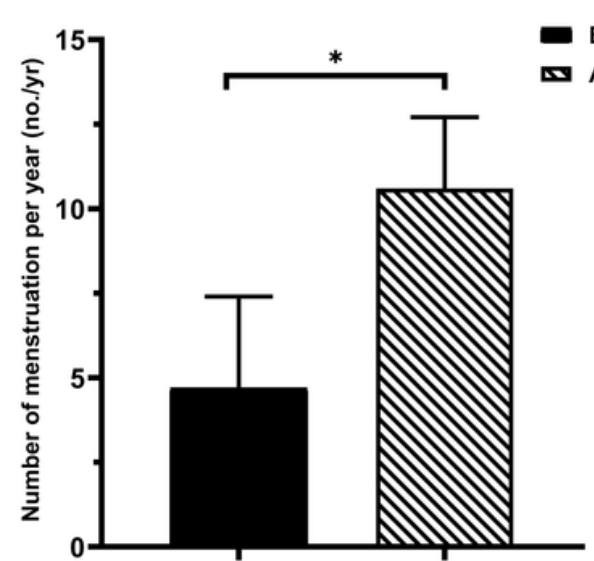

A

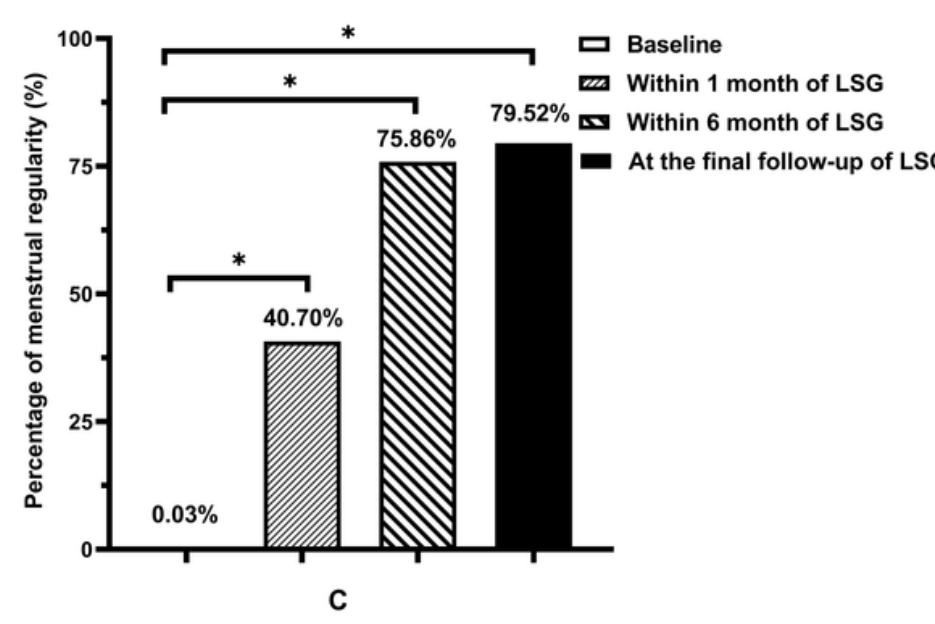

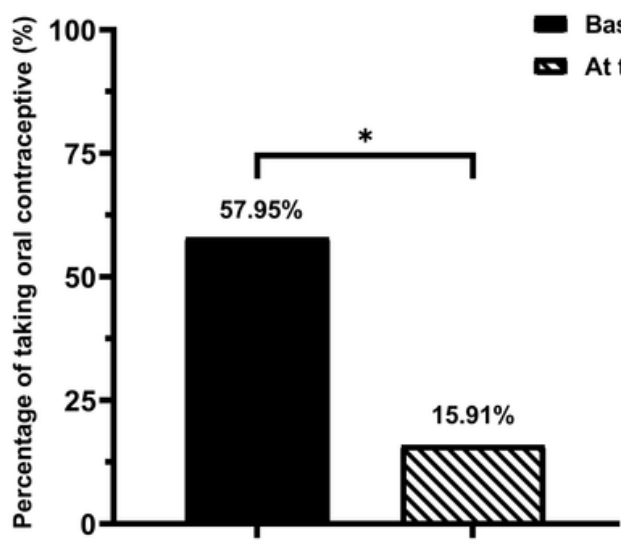

B

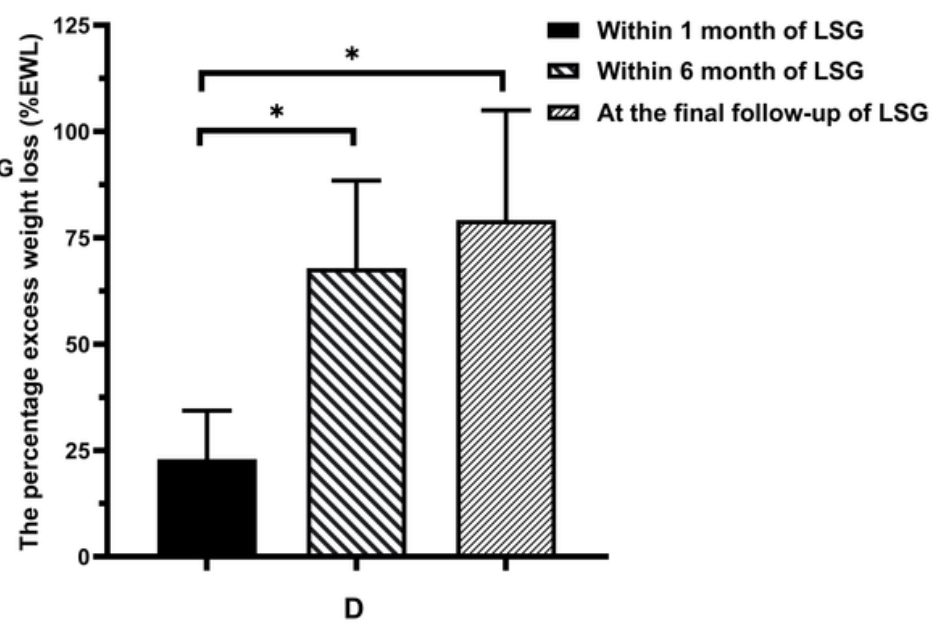

Figure 2

Menstrual status, BMI and use of oral contraceptives in PCOS women with obesity before and after LSG, *means $\mathrm{P}<0.05$.
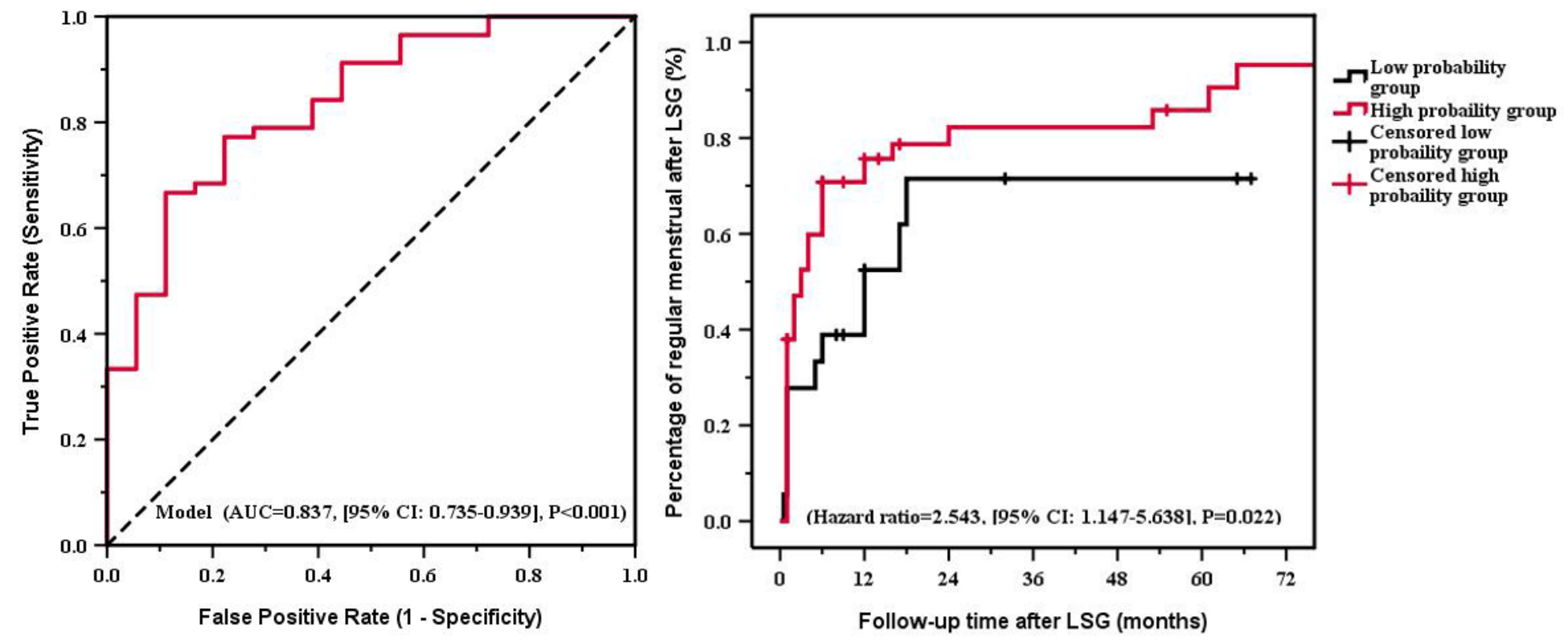


\section{Figure 3}

Receiver operating characteristic and Kaplan-Meier curve for predicting the recovery of regular menstrual cycles within 6 months after LSG in the PCOS group. A. The predictive model combined four clinical indicators, including duration of PCOS, number of menstruations per year, homeostatic model assessment of insulin resistance, and total testosterone and the possible confounders (after adjusting age, body mass index and alanine aminotransferase). B. Kaplan-Meier curves were calculated according to the optimal cut-off point for the combined predictive indicator in the predictive model. PCOS women with obesity were categorized into the high probability group and the low probability group. 